\title{
New Isoflavone Derivative and Other Flavonoids from the Resin of Amburana cearensis
}

\author{
Paulo N. Bandeira, ${ }^{*, a}$ Silvana S. de Farias, ${ }^{a}$ Telma L. G. Lemos, ${ }^{b}$ Raimundo Braz-Filho, ${ }^{c}$ \\ Hélcio S. Santos, ${ }^{a}$ Maria R. J. R. Albuquerque ${ }^{a}$ and Sônia M. O. Costa ${ }^{d}$ \\ ${ }^{a}$ Laboratório de Química Orgânica, Universidade Estadual Vale do Acaraú, \\ 62040-370 Sobral-CE, Brazil \\ ${ }^{b}$ Departamento de Química Orgânica e Inorgânica, Universidade Federal do Ceará, \\ 60451-970 Fortaleza-CE, Brazil \\ 'Laboratório de Ciências Químicas, CCT, Universidade Estadual do Norte Fluminense Darcy \\ Ribeiro, 28013-602 Campos-RJ, Brazil \\ ${ }^{d}$ Departamento de Física e Química, Universidade Estadual do Ceará, \\ 60749-000 Fortaleza-CE, Brazil
}

\begin{abstract}
A investigação fitoquímica da resina de Amburana cearensis A. C. Smith permitiu o isolamento de um novo composto: 3',4'-dimetóxi-1'-(7-metóxi-4-oxo-4H-cromen-3-il) benzo-2',5'-quinona (1), juntamente com seis compostos conhecidos e identificados como: 4,2',4'-triidroxichalcona (2), 7,8,3',4'-tetrametoxiisoflavona (3), 4,2',4'-triidroxi-3metoxichalcona (4), 3,4,5-trimetoxicinamaldeido (5), 3',4'-dimetoxi-7-hidroxiisoflavona (6) e 6,7,4'-trimetoxi-3'-hidroxiisoflavona (7). As estruturas foram estabelecidas com base na análise dos dados espectrométricos de: IV, EI-EM, HR-ESI-EM e RMN incluindo experimentos 2D.
\end{abstract}

Phytochemical investigation of the resin of Amburana cearensis A. C. Smith allowed the isolation of a new compound: 3',4'-dimethoxy-1'-(7-methoxy-4-oxo-4Hcromen-3-yl)benzo-2',5'-quinone (1), together with six known compounds identified as: 4,2',4'-trihydroxychalcone (2), 7,8,3',4'-tetramethoxyisoflavone (3), 4,2',4'-trihydroxy-3methoxychalcone (4), 3,4,5-trimethoxycinnamaldehyde (5), 3',4'-dimethoxy-7-hydroxyisoflavone (6) and 6,7,4'-trimethoxy-3'-hydroxyisoflavone (7). The structures were established from the IR, HR-ESI-MS and NMR spectral data, including 2D-NMR experiments.

Keywords: Amburana cearensis, resin, phytochemical investigation, flavonoids

\section{Introduction}

Amburana cearensis A. C. Smith (Sin. Torrensea cearensis Fr. AlI), Leguminoseae family, Papilionoideae (Fabaceae), is a tree popularly known as "imburana de cheiro", "cerejera" and "cumaru" in northeast of Brazi1.,2 The syrup of trunk bark is used in folk medicine for treatment of respiratory diseases as cold, bronchits and asthma. Pharmacological studies using hydroalcoolic syrup from the bark trunk showed anti-inflammatory, analgesic, bronchodilator and antinociceptive activities. ${ }^{3-6}$ Recent studies $^{7}$ have shown that amburoside A, isokaempferide,

*e-mail: bandeirapn@yahoo.com.br kaempferol and polyphenols isolated from the trunk bark of A. cearensis also showed present neuroprotective, antioxidant, antiinflamatory, anticarcinogenic and hepatoprotective activities.

Previous studies with the bark from A. cearensis resulted in the isolation of 4-(O- $\beta$-D-glucopyranosyl)hydroxy-7-(3',4'-dihydroxy-benzoyl)-benzyl, 4-(O- $\beta-D$ glucopyranosyl)-hydroxy-7-(3'-methoxy-4'-hydroxybenzoyl)-benzyl, coumarin, sucrose, vanilic acid, protocatechuic acids, afrormosin, isokaempferide, kaempferol, quercetin, 4' -methoxy-fisetin, amburoside A and a mixture of $\beta$-sitosterol and stigmasterol glucoside). ${ }^{8,9}$ Phytochemical investigation was carried out recently with especies obtained from seed germinations. ${ }^{10}$ 
This paper describes the chemical investigation of the resin of A. cearensis, which resulted in the isolation of a new compound named as 3', 4'-dimethoxy-l'-(7-methoxy4-oxo-4H-cromen-3-yl)benzo-2', 5' -quinone (1).

\section{Experimental}

\section{General procedure}

The melting point was determined using a Mettler Toledo FP82HT micromelting point apparatus. The IR spectra were measured in $\mathrm{KBr}$ pellets, using Perkin-Elmer, FT-IR Spectrum 1000. All the NMR data were recorded using a Bruker Avance DPX 300 and Avance DRX-500 spectrometer operating in the frequency of the hydrogen at 300.13 and $500.13 \mathrm{MHz}$ in the frequency of the carbon for 75.47 and $125.75 \mathrm{MHz}$. The spectra were recorded in $\mathrm{CDCl}_{3} \mathrm{C}_{5} \mathrm{D}_{5} \mathrm{~N}$ and $\mathrm{CD}_{3} \mathrm{OD}$. High resolution data were obtained in a MS-IT-TOF mass spectrometer.

\section{Plant material}

The resin of A. cearensis was collected in city of Limoeiro do Norte, Ceará, Brazil. The plant material was identified in the Departamento de Biologia do Centro de Ciências da Universidade Federal do Ceará, Brazil. A voucher (No. 847) specimen is deposited in the Herbarium Prisco Bezzera of this University.

\section{Extraction and isolation}

The resin $(400 \mathrm{~g})$ was submitted to an extraction with $\mathrm{EtOH}$ at room temperature and yielded (23.46 g). The EtOH extract was dissolved in $200 \mathrm{~mL}$ of water and partitioned in the $\mathrm{CHCl}_{3}$ and EtOAc yielded $(8.1 \mathrm{~g})$ and $(0.7 \mathrm{~g})$ respectively. The EtOAc fraction $(0.7 \mathrm{~g})$ was subjected to $\mathrm{CC}$ on $\mathrm{SiO}_{2}$ using EtOAc, $\mathrm{CHCl}_{3}$ and $\mathrm{MeOH}$. The $\mathrm{CHCl}_{3}$ fraction $(0.15 \mathrm{~g})$ was chromatographed in sephadex LH-20 column using $\mathrm{MeOH}$, obtaining compound $\mathbf{1}(18 \mathrm{mg})$ and $\mathbf{2}(30 \mathrm{mg})$. The $\mathrm{CHCl}_{3}$ fraction (8.1 g) was subjected to $\mathrm{CC}$ on $\mathrm{SiO}_{2}$ using EtOAc, $\mathrm{CHCl}_{3}$ and $\mathrm{MeOH}$. The $\mathrm{CHCl}_{3}$ fraction $(0.30 \mathrm{~g})$ was successively chromatographed in sephadex LH-20 column using $\mathrm{MeOH}$ and obtaining compound 3 (40 mg), 4 (23 mg), $5(15 \mathrm{mg}), \mathbf{6}(10 \mathrm{mg})$ and $\mathbf{7}(16 \mathrm{mg})$.

3',4'-Dimethoxy-1'-(7-methoxy-4-oxo-4H-cromen-3-yl) benzo-2',5'-quinone (1)

Orange solid, mp 196-198 ${ }^{\circ} \mathrm{C}$; IR (KBr) $v_{\max } / \mathrm{cm}^{-1}: 1649$, 1628, 1595, 1439, 1279, 1100, 835; ${ }^{1} \mathrm{H}$ and ${ }^{13} \mathrm{C}$ NMR (see Table 1); HR-ESI-MS spectrum revealed formation of molecular ions $m / z 343.0931\left(\mathrm{C}_{18} \mathrm{H}_{14} \mathrm{O}_{7}+\mathrm{H}^{+}\right), 365.0661$

Table 1. ${ }^{1} \mathrm{H}$ NMR $(500 \mathrm{MHz})$ and ${ }^{13} \mathrm{C}$ NMR $(125 \mathrm{MHz})$ data of $\mathbf{1}$ (in $\mathrm{CDCl}_{3}$ ) compared with values of $\mathbf{8}$ (DMSO- $d_{6}$, (dd, ppm), $J$ in $\mathrm{Hz} *$

\begin{tabular}{|c|c|c|c|c|c|c|}
\hline \multicolumn{5}{|c|}{1} & \multicolumn{2}{|c|}{8} \\
\hline & \multicolumn{2}{|c|}{${ }^{1} \mathrm{H},{ }^{13} \mathrm{C}-\mathrm{HMQC}$} & \multicolumn{2}{|c|}{${ }^{1} \mathrm{H},{ }^{13} \mathrm{C}-\mathrm{HMBC}$} & \multirow[b]{2}{*}{$\delta(\mathrm{C})$} & \multirow[b]{2}{*}{$\delta(\mathrm{H})$} \\
\hline & $\delta(\mathrm{C})$ & $\delta(\mathrm{H})$ & ${ }^{2} J$ & ${ }^{3} J$ & & \\
\hline$\overline{C(2)}$ & 156.5 & $8.16(\mathrm{~s})$ & & & 157,5 & $8.36(\mathrm{~s})$ \\
\hline $\mathrm{C}(3)$ & 116.3 & & $\mathrm{H}-2$ & H-6 & 115.5 & \\
\hline $\mathrm{C}(4)$ & 174.4 & & & H-2; H-5 & 178.8 & \\
\hline $\mathrm{C}(5)$ & 127.9 & $8.15(\mathrm{~d}, J 8.9)$ & & & 161.6 & \\
\hline$C(6)$ & 115.1 & $7.02(\mathrm{dd}, J 8.9,2.3)$ & & H-8 & 99.5 & $6.44(\mathrm{~d}, J 1.5)$ \\
\hline $\mathrm{C}(7)$ & 165.0 & & H-6; H-8 & $\mathrm{MeO}-7$ & 164.7 & \\
\hline $\mathrm{C}(8)$ & 100.4 & $6.88(\mathrm{~d}, J(2.3)$ & & H-6 & 94.1 & $6.27(\mathrm{~d}, J 1.5)$ \\
\hline $\mathrm{C}(9)$ & 158.0 & & $\mathrm{H}-8$ & $\mathrm{H}-2$ & 157.1 & \\
\hline $\mathrm{C}(10)$ & 118.0 & & & H-6; H-8 & 104.0 & \\
\hline $\mathrm{C}\left(1^{\prime}\right)$ & 135.0 & & H-6 & $\mathrm{H}-2$ & 137.9 & \\
\hline $\mathrm{C}\left(2^{\prime}\right)$ & 183.9 & & & H-6'; MeO-2' & 185.0 & \\
\hline$C\left(3^{\prime}\right)$ & 145.0 & & & $\mathrm{MeO}-3$ & 107.9 & $6.26(\mathrm{~s})$ \\
\hline $\mathrm{C}\left(4^{\prime}\right)$ & 158.2 & & & MeO-4'; H-6' & 158.4 & \\
\hline $\mathrm{C}\left(5^{\prime}\right)$ & 183.9 & & & & 181.4 & \\
\hline$C\left(6^{\prime}\right)$ & 133.9 & $7.17(\mathrm{~s})$ & & & 133.2 & $7.05(\mathrm{~s})$ \\
\hline $\mathrm{MeO}-7$ & 55.9 & $3.93(\mathrm{~s})$ & & & & \\
\hline $\mathrm{MeO}-3$ ' & 61.2 & $4.03(\mathrm{~s})$ & & & & \\
\hline $\mathrm{MeO}-4$ ' & 61.5 & $4.08(\mathrm{~s})$ & & & 56.3 & $3.83(\mathrm{~s})$ \\
\hline
\end{tabular}

*Number of hydrogens bound to carbon atoms deduced by comparative analysis of ${ }^{1} \mathrm{H}$ and DEPT ${ }^{13} \mathrm{C}$ NMR spectra. Chemical shifts and coupling constants $(J)$ were obtained of $1 \mathrm{D}^{1} \mathrm{H}$ NMR spectrum. ${ }^{1} \mathrm{H},{ }^{1} \mathrm{H}$ COSY and ${ }^{1} \mathrm{H},{ }^{1} \mathrm{H}$ NOESY experiments were also used for these assignments in compared with literature data 8 (ref. 13). 
$\left(\mathrm{C}_{18} \mathrm{H}_{14} \mathrm{O}_{7}+\mathrm{Na}^{+}\right)$and $381.0438\left(\mathrm{C}_{18} \mathrm{H}_{14} \mathrm{O}_{7}+\mathrm{K}^{+}\right)$. LR-EIMS $m / z$ (rel; Int.): ([M+], 94.5\%), $200\left[\mathrm{M}^{+}-142\right] 100 \%$, $150\left[\mathrm{M}^{+}-193\right] 32.9 \%, 327\left[\mathrm{M}^{+}-15\right] 8.2 \%$.

\section{Results and Discussion}

Compound 1 was obtained as orange solid. The HR-ESIMS spectrum showed a pseudo molecular íons peaks at $\mathrm{m} / \mathrm{z}$ $343.0931\left(\mathrm{C}_{18} \mathrm{H}_{14} \mathrm{O}_{7}+\mathrm{H}^{+}\right.$, requires 343.0818), 365.0661 $\left(\mathrm{C}_{18} \mathrm{H}_{14} \mathrm{O}_{7}+\mathrm{Na}^{+}\right.$, requires 365.0637) and 381.0438 $\left(\mathrm{C}_{18} \mathrm{H}_{14} \mathrm{O}_{7}\right.$ $+\mathrm{K}^{+}$, requires 381.0377). The IR spectrum exhibited bands at 1650,1628 and $1595\left(\mathrm{v}_{\mathrm{c}=0}\right) \mathrm{cm}^{-1}$ suggesting the presence of three conjugated carbonyl groups. ${ }^{11,12}$ Comparative analysis of the ${ }^{1} \mathrm{H}$ and DEPT ${ }^{13} \mathrm{C}$ NMR spectra (Table 1) allowed to recognize signals for three methoxyl groups and fifteen $\mathrm{sp}^{2}$ carbons: five methines (including one linked to oxygen atom at $\delta_{\mathrm{c}} 156.5$, compatible with $\mathrm{CH}-2$ of isoflavones) and ten non-hydrogenated [including three carbonyl groups at $\delta_{c} 174.4(\mathrm{C}-4), \delta_{c} 183.9$ (C-2' and C-5') and four linked to oxygen atoms: $\delta_{\mathrm{c}} 165.0(\mathrm{C}-7), \delta \mathrm{c} 158.0(\mathrm{C}-9), \delta \mathrm{c} 145.0$
(C-3') and $\left.\delta_{c} 158.2\left(\mathrm{C}^{\prime} 4^{\prime}\right)\right]$. The signals observed in the ${ }^{13} \mathrm{C}$ NMR spectra at $\delta_{\mathrm{c}} 183.9$ and 174.4 were attributed to carbonyl groups at the 1,4-benzoquinone and flavonoid moieties, ${ }^{13}$ respectively. Furthermore, the ${ }^{1} \mathrm{H}$ NMR exhibited a characteristic a singlet at $\delta_{\mathrm{H}} 8.16$, assigned to $\mathrm{H}-2$ of an isoflavone, confirmed by HMQC spectrum by the presence of a transversal peak corresponding to direct correlation of this hydrogen signal with ${ }^{13} \mathrm{C}$ signal of methine at $\delta_{\mathrm{c}} 156.5$ $(\mathrm{CH}-2)$, and at $\delta_{\mathrm{H}} 3.93, \delta_{\mathrm{H}} 4.03$ and $\delta_{\mathrm{H}} 4.08$, corresponding to three methoxyl groups located at C-7, C-3' and C-4', respectively. The location of these methoxyl groups was observed at the heteronuclear long range correlations in the HMBC spectrum (Table 1), which showed correlations of the methoxyl signal at $\delta_{\mathrm{H}} 3.93$ with C-7 $\left(\delta \mathrm{c} 165.0,{ }^{3} J_{\mathrm{CH}}, \delta_{\mathrm{H}}\right.$ $4.03\left(\mathrm{MeO}-3\right.$ ') with $\delta_{\mathrm{c}} 145.0\left(\mathrm{C}-3{ }^{\prime},{ }^{3} J_{\mathrm{CH}}\right)$ and $\delta_{\mathrm{H}} 4.08(\mathrm{MeO}-$ 4') with $\delta_{\mathrm{c}} 158.2\left(\mathrm{C}-4\right.$ ', $\left.{ }^{3} J_{\mathrm{CH}}\right)$. The presence of a methoxyl group at $\mathrm{C}-7$ was additionally confirmed by nOe effects observed in the ${ }^{1} \mathrm{H},{ }^{1} \mathrm{H}$ NOESY spectrum, which revealed spatial interactions with the hydrogen atoms H-6 and H-8. The multiplicities of these signals of hydrogen atoms H-6

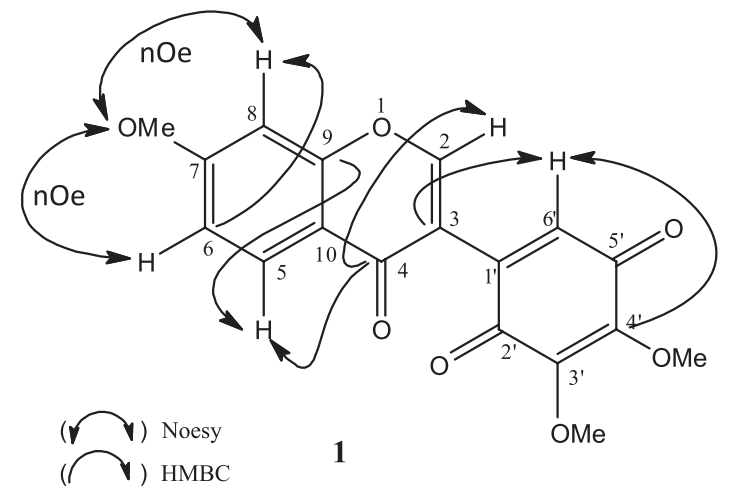<smiles>COc1cc(/C=C/C=O)cc(OC)c1OC</smiles><smiles>[R]c1cc(/C=C/C(=O)c2ccc(O)cc2O)ccc1O</smiles><smiles>[R]c1cc(-c2c(C)oc3c([R])c([R])c([R])cc3c2=O)ccc1OC</smiles><smiles>[R][R]([R4])OC</smiles>

$3 \mathrm{R}=\mathrm{R}^{1}=\mathrm{R}^{2}=$ OMe, $\mathrm{R}^{3}=\mathrm{H}$

$6 \mathrm{R}=\mathrm{OH}, \mathrm{R}^{1}=\mathrm{R}^{3}=\mathrm{H}, \mathrm{R}^{2}=\mathrm{OMe}$

$7 \mathrm{R}=\mathrm{R}^{3}=\mathrm{OMe}, \mathrm{R}^{1}=\mathrm{H}, \mathrm{R}^{2}=\mathrm{OH}$

Figure 1. Structure of the chemical constituents (1-7) isolated of resin of Amburana cearensis A.C Smith and the compound 8 from reference 13. 
$\left(\delta_{\mathrm{H}} 7.02\right.$, dd, $J 8.9$ and $\left.2.3 \mathrm{~Hz}\right)$ and $\mathrm{H}-8\left(\delta_{\mathrm{H}} 6.88, \mathrm{~d}, J 2.3 \mathrm{~Hz}\right)$ in combination with the corresponding $\mathrm{H}-5\left(\delta_{\mathrm{H}} 8.15\right.$, d, $J 8.9 \mathrm{~Hz}$ ), correlated via one bond in the HMQC spectrum with the ${ }^{13} \mathrm{C}$ signal at 127.9 , allowed to define the ring $\mathrm{A}$, sustaining a methoxyl group at carbon atom C-7. The HMBC spectrum was also used to characterize the presence of a 2,3-dimethoxy-1,4- benzoquinone moiety at $\mathrm{C}-3$ through heteronuclear long range correlation (Table 1) of the carbon C-4' $\left(\delta_{\mathrm{c}} 158.2\right)$, with both H-6' $\left(\delta_{\mathrm{H}} 7.17, \mathrm{~s}^{3}{ }^{3} \mathrm{CH}_{\mathrm{CH}}\right)$ and MeO-4' $\left(\delta_{\mathrm{H}} 4.08, \mathrm{~s},{ }^{3} J_{\mathrm{CH}}\right), \mathrm{C}-3{ }^{\prime}\left(\delta_{\mathrm{c}} 145.0\right)$ with MeO-3' $\left(\delta_{\mathrm{H}} 4.03, \mathrm{~s}\right.$, $\left.{ }^{3} J_{\mathrm{CH}}\right), \mathrm{C}-1$ ' $\left(\delta_{\mathrm{H}} 135.0\right)$ with both H-2 $\left(\delta_{\mathrm{H}} 8.16, \mathrm{~s},{ }^{3} J_{\mathrm{CH}}\right)$ and H-6 $\left(\delta_{\mathrm{H}} 7.17, \mathrm{~s},{ }^{2} J_{\mathrm{CH}}\right)$ and C-3 $\left(\delta_{\mathrm{c}} 116.3\right)$ with both H-2 $\left(\delta_{\mathrm{H}}\right.$ $\left.8.16, \mathrm{~s},{ }^{2} J_{\mathrm{CH}}\right)$ and $\mathrm{H}-6{ }^{\prime}\left(\delta_{\mathrm{H}} 7.17, \mathrm{~s},{ }^{3} J_{\mathrm{CH}}\right)$. Additional analysis of the ${ }^{13} \mathrm{C}$ NMR spectra of 1 revealed, the ${ }^{13} \mathrm{C}$ chemical shifts $\left(\delta_{\mathrm{c}} 145.0\right)$ for the $\mathrm{C}-3$ ' and $\left(\delta_{\mathrm{c}} 158.2\right)$ for the $\mathrm{C}-4$ ' and for the C-2'/C-5' $\left(\delta_{\mathrm{c}} 183.9\right)$, whereby after comparison of these said data with the correspondent values, $\delta_{\mathrm{c}} 107.9$ $\left(\mathrm{CH}-3^{\prime}\right), \delta_{\mathrm{c}} 158.4\left(\mathrm{C}^{\prime} 4^{\prime}\right), \delta_{\mathrm{c}} 185.0\left(\mathrm{C}-2^{\prime}\right)$ and $\delta_{\mathrm{c}} 181.4\left(\mathrm{C}-5^{\prime}\right)$, described in the literature, ${ }^{13}$ suggests the presence of an additional methoxyl group at carbon C-3' of $\mathbf{1}$ as differences observed between spectral data of $\mathbf{1}$ and of $\mathbf{8}$ (Table 1). The base peak at $\mathrm{m} / z 200(100 \%)$ observed in the LR-EI-MS, significantly contributed to characterize and to locate the 2,3-dimethoxy-1,4- benzoquinone moiety at C-3 (Figure 1).

Therefore, the structure of compound $\mathbf{1}$ was assigned as a new isoflavone and it is characterized as 3', 4'dimethoxy-1'-(7 -methoxy-4-oxo-4H-cromen-3-yl)-benzo2',5'-quinone.

The compounds $\mathbf{2}, \mathbf{3}, \mathbf{4}, \mathbf{5}, \mathbf{6}$ and $\mathbf{7}$ were identified through spectroscopic data compared with literature data as: 4,2',4'-trihydroxychalcone, ${ }^{14} 7,8,3^{\prime}, 4^{\prime}$ '-tetrarnethoxyisoflavone, ${ }^{15} 4,2^{\prime}, 4^{\prime}$-trihydroxy-3-methoxychalcone, ${ }^{16}$ 3,4,5- trimethoxycinnamaldehyde, ${ }^{17} 3^{\prime}, 4^{\prime}$ '-dimethoxy-7hydroxyisoflavone ${ }^{18}$ and 6,7,4'-trimethoxy- 3'-hydroxyisoflavone ${ }^{19}$ (Figure 1), respectively.

\section{Supplementary Information}

Supplementary data are available free of charge at http://jbcs.sbq.org.br, as PDF file.

\section{Acknowledgments}

The authors whishes to thank CNPq/FUNCAP for financial support, CENAUREMN by NMR spectra and LEMANOR by mass spectrum.

\section{References}

1. Corrêa, M. P.; Dicionário das Plantas Úteis do Brasil e das Exóticas Cultivadas, Ministério da Agricultura: Brasília, 1984.
2. Maia, G. N.; Caatinga: Árvores e Arbustos e Suas utilidades, D \& Z ed.: São Paulo, Brasil, 2004.

3. Leal, L. K. A. M.; Matos, M. E.; Matos, F. J. A.; Ribeiro, R. A.; Ferreira, F. V.; Viana, G. S. B.; Phytomedicine 1997, 4, 221.

4. Leal, L. K. A. M.; Nechio, M.; Silveira, E. R.; Canuto, K. M.; Fontenele, J. B.; Ribeiro, R. A.; Viana, G. S. B.; Phytother. Res. 2003, 17, 335 .

5. Oliveira, R. R. B.; Góis, R. M. O.; Siqueira, R. S.; Almeida, J. R. G. S.; Lima, J. T. L.; Nunes, X. P.; Oliveira, V. R.; Siqueira, J. S.; Quintons-Junior, L. J.; Braz. J. Pharmcog. 2009, 19, 672.

6. Leal, L. K. A. M.; Ferreira, A. A. G.; Bezerra, G. A.; Matos, F. J. A.; Viana, G. S. B.; J. Ethnopharmacol. 2000, 70, 151.

7. Leal, L. K. A. M.; Canuto, K. M.; Costa, K. C. S.; NobreJunior, H. V.; Vasconcelos, S. M.; Silveira, E. R.; Ferreira, M. V. P.; Fontenele, J. B.; Andrade, G. M.; Viana, G. S. B.; Basic Clin. Pharmacol. Toxicol. 2008, 104, 198; Leal, L. K. A. M.; Nobre-Junior, H. V.; Cunha, G. M. A.; Moraes, M. O.; Pessoa, C.; Oliveira, R. A.; Silveira, E. R.; Canuto, K. M.; Viana, G. S. B.; Neurosci. Lett. 2005, 388, 86; Leal, L. K. A. M.; Costa, M. F.; Pitombeira, M.; Barroso, V. M.; Silveira, E. R.; Canuto, K. M.; Viana, G. S. B.; Life Sci. 2006, 79, 98; Costa-Lotufo, L. V.; Jimenez, P. C.; Wilke, D. V.; Leal, L. K. A. M.; Cunha, G. M. A.; Silveira, E. R.; Canuto, K. M.; Viana, G. S. B.; Moraes, M. E. A,; de Moraes, M. O.; Pessoa, C.; J. Biosci. 2003, 58, 9; Leal, L. K. A. M.; Fonseca, F. N.; Pereira, F. A.; Canuto, K. M.; Felipe, C. F. B.; Fontenele, J. B.; Pitombeira, M. V.; Silveira, E. R.; Viana, G. S. B.; Planta Med. 2008, 74, 497.

8. Bravo, J. A. B.; Sauvain, M.; Gimenez, A.; Muñoz, V.; Callapa, J.; Le Men-Oliver, L.; Massiot, G.; Lavaud, C.; Phytochemistry 1999, 50, 71.

9. Canuto, K. M.; Silveira, E. R.; Quim. Nova 2006, 29, 1241.

10. Canuto, K. M.; Silveira, E. R.; Bezerra, A. M. E.; Quim. Nova 2010, 33, 662.

11. Silverstein, R. M.; Webster, F. X.; Identificação Espectrométrica de Compostos Orgânicos, $6^{\text {th }}$ ed., LTC, ed.: Rio de Janeiro, 2000.

12. Marques, W. B.; Santos, H. S.; Pessoa, O. D. L.; Braz-Filho, R.; Lemos, T. L.G.; Phytochemistry 2000, 55, 793.

13. Hamburger, M. O.; Cordell, G. A.; J. Nat. Prod. 1987, 50, 696.

14. Markham, K. R.; Ternai, B.; Tetrahedron 1976, 32, 2607.

15. Rao, V.; Murthy, S. R.; Ward, R. T.; Phytochemistry 1984, 23, 1493.

16. Nielsen, S. F.; Christensen, S. B.; Cruciani, G.; Kharazmi, A.; Liljefors, T.; J. Med. Chem. 1998, 41, 4819.

17. Mohammad, I.; Watterman, P. G.; J. Nat. Prod. 1985, 48, 328.

18. Rong, H.; Stevens, J. F.; Deizer, M. L.; De Cooman, L.; De Keukeleire, D.; Planta Med. 1998, 64, 620.

19. Razdan, T. K.; Kachroo, P. K.; Qadri, A. K.; Kalla, A. K.; Taneja, S. K.; Dhar, K. L.; Phytochemistry 1996, 41, 947.

Submitted: November 21, 2009 Published online: October 7, 2010 


\section{New Isoflavone Derivative and others Flavonoids from the Resin of Amburana cearensis}

Paulo N. Bandeira, ${ }^{*, a}$ Silvana S. de Farias, ${ }^{a}$ Telma L. G. Lemos, ${ }^{b}$ Raimundo Braz-Filho, ${ }^{c}$ Hélcio S. Santos, ${ }^{a}$ Maria R. J. R. Albuquerque ${ }^{a}$ and Sônia M. O. Costa ${ }^{d}$

${ }^{a}$ Laboratório de Química Orgânica, Universidade Estadual Vale do Acaraú, 62040-370 Sobral-CE, Brazil

${ }^{b}$ Departamento de Química Orgânica e Inorgânica, Universidade Federal do Ceará, 60451-970 Fortaleza-CE, Brazil

${ }^{c}$ Laboratório de Ciências Químicas, CCT, Universidade Estadual do Norte Fluminense Darcy Ribeiro, 28013-602 Campos-RJ, Brazil

${ }^{d}$ Departamento de Física e Química, Universidade Estadual do Ceará, 60749-000 Fortaleza-CE, Brazil

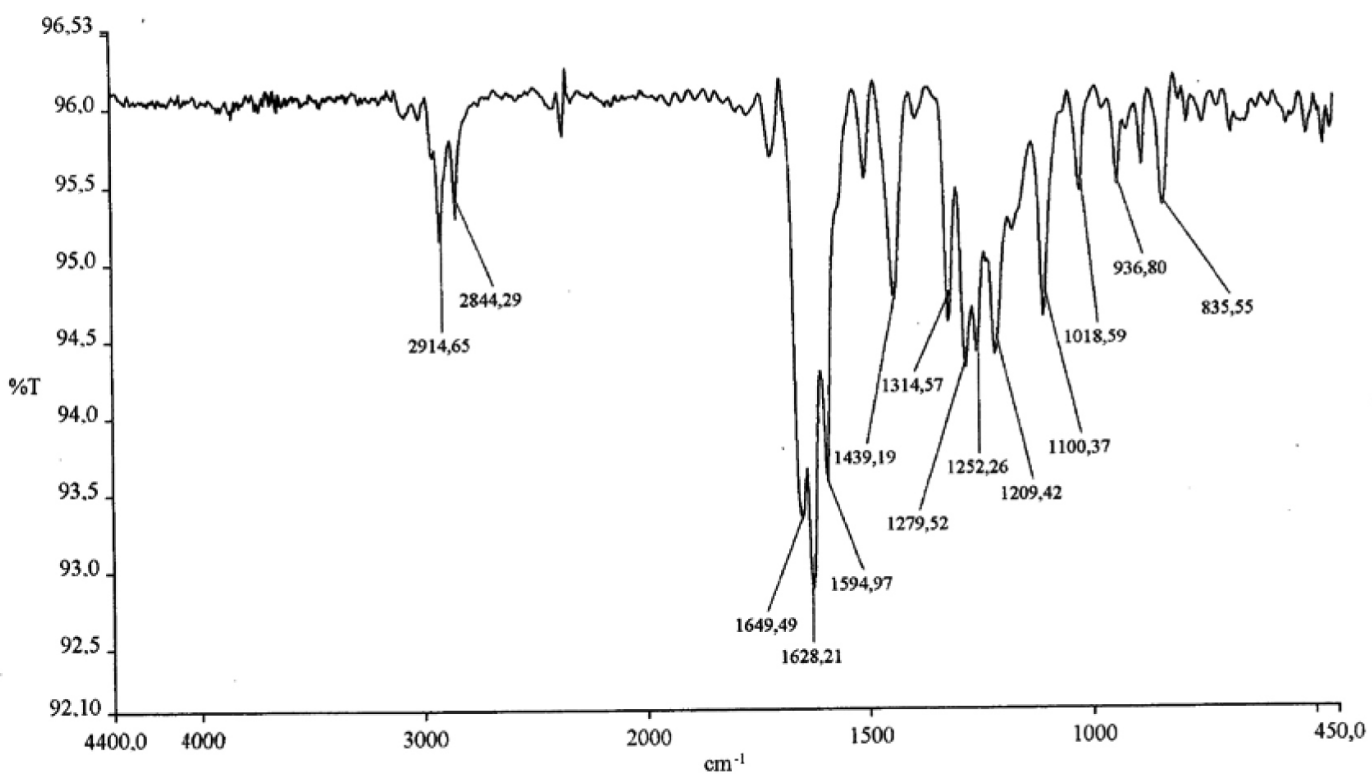

Figure S1. Infrared spectrum of compound $\mathbf{1}$ ( $\mathrm{KBr}$ pellets). 


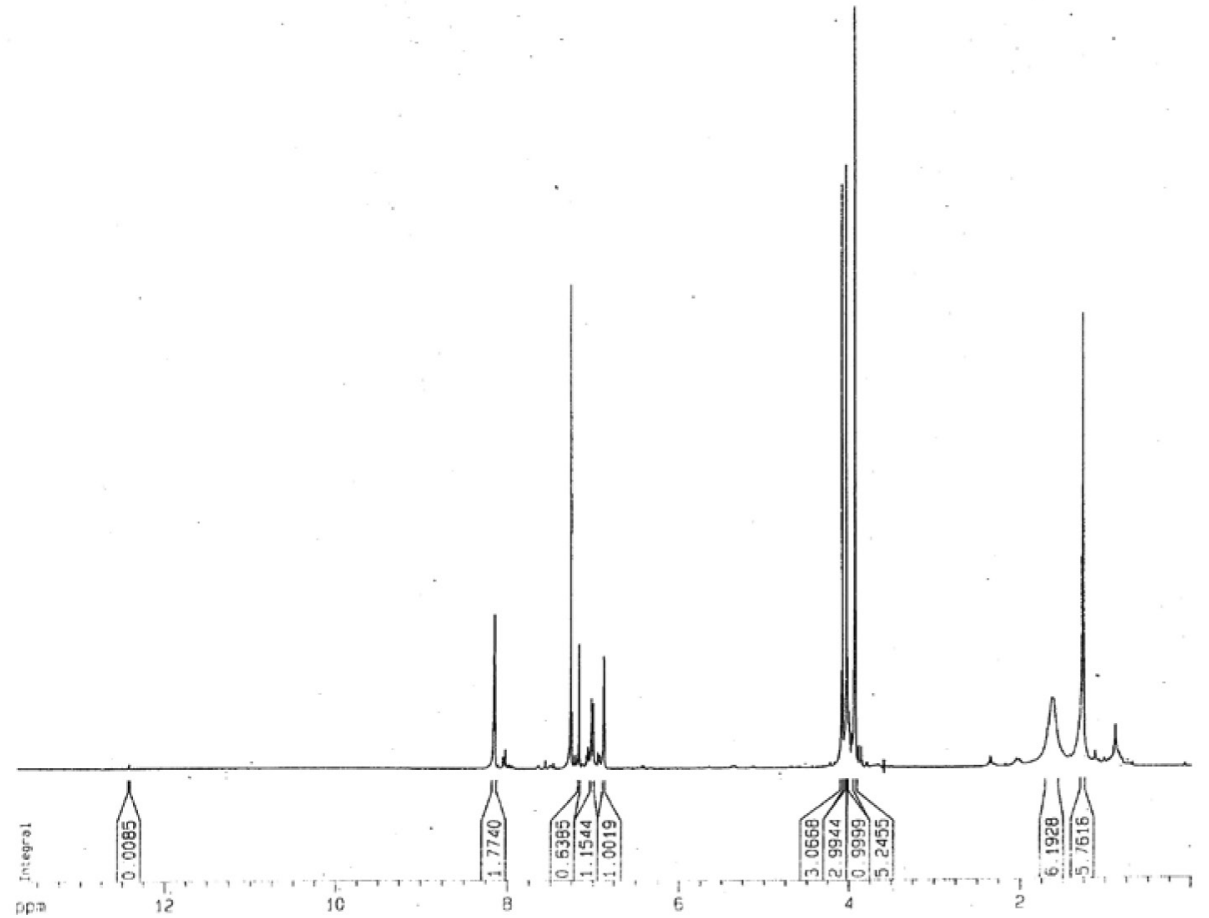

Figure S2. ${ }^{1} \mathrm{H}$ NMR spectrum of $\mathbf{1}\left(500 \mathrm{MHz}, \mathrm{CDCl}_{3}\right)$.

형

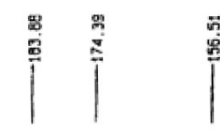

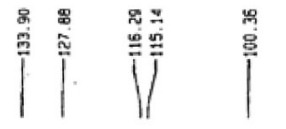

U

$\underset{\sim}{\sim}$

|

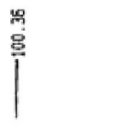

1

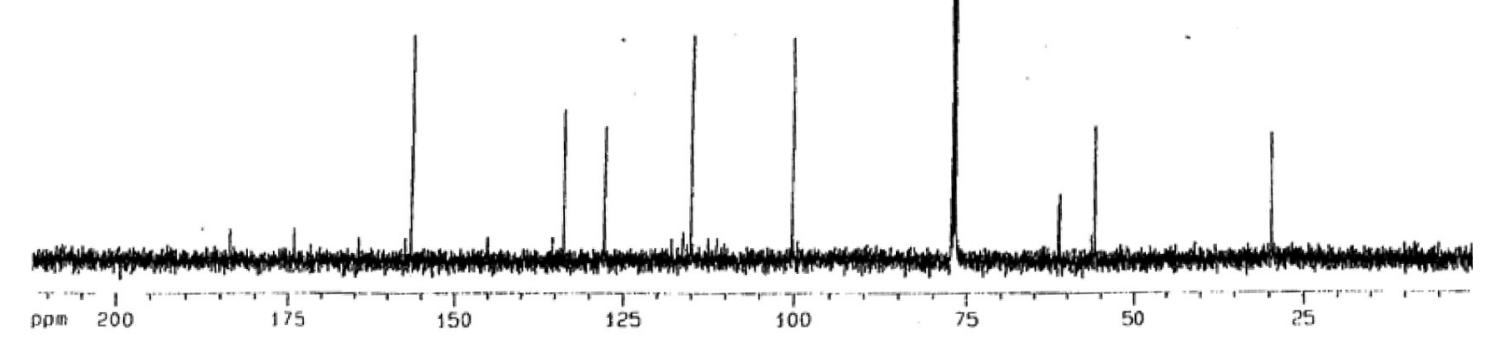

Figure S3. ${ }^{13} \mathrm{C}$ NMR spectrum of $\mathbf{1}\left(125 \mathrm{MHz}, \mathrm{CDCl}_{3}\right)$. 
Vol. 22, No. 2, 2011

Bandeira et al.

S3
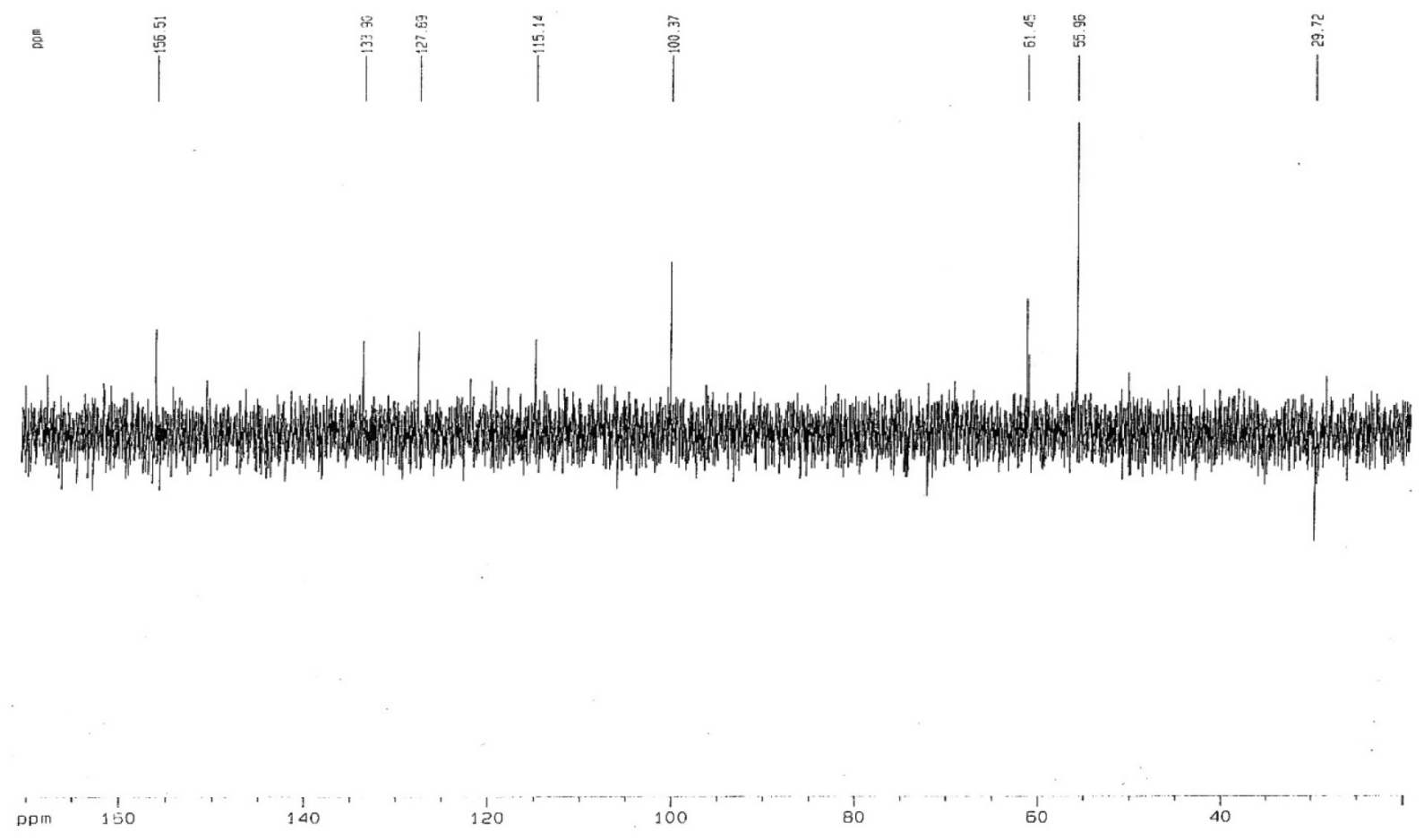

Figure S4. ${ }^{13} \mathrm{C}$ NMR-DEPT $135^{\circ}$ of $\mathbf{1}$.

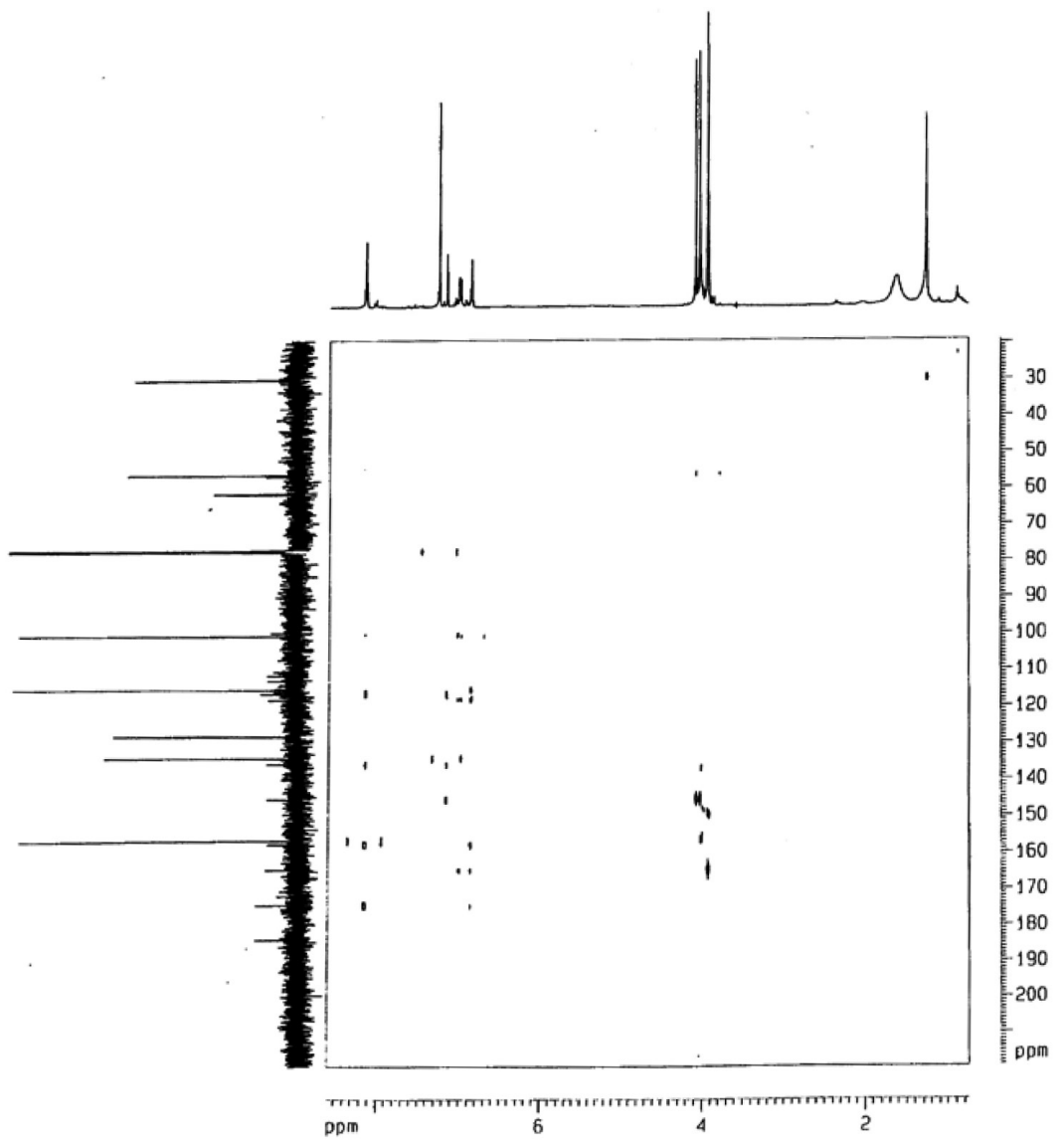

Figure S5. ${ }^{1} \mathrm{H},{ }^{13} \mathrm{C}$ HMBC-NMR spectrum of $\mathbf{1}\left(500 \mathrm{MHz}, \mathrm{CDCl}_{3}\right)$. 


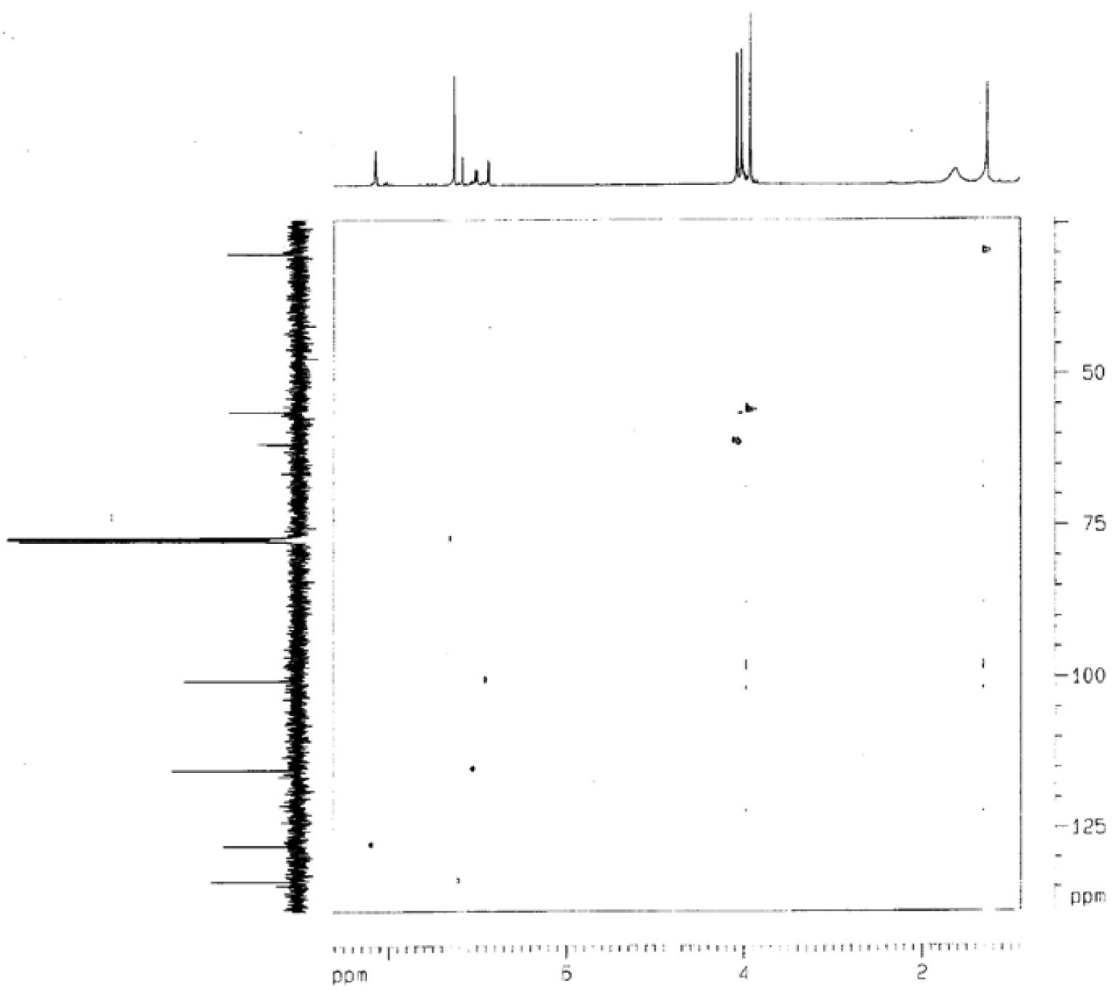

Figure S6. ${ }^{1} \mathrm{H},{ }^{13} \mathrm{C}$ HMQC-NMR of $\mathbf{1}\left(500 \mathrm{MHz}, \mathrm{CDCl}_{3}\right)$.

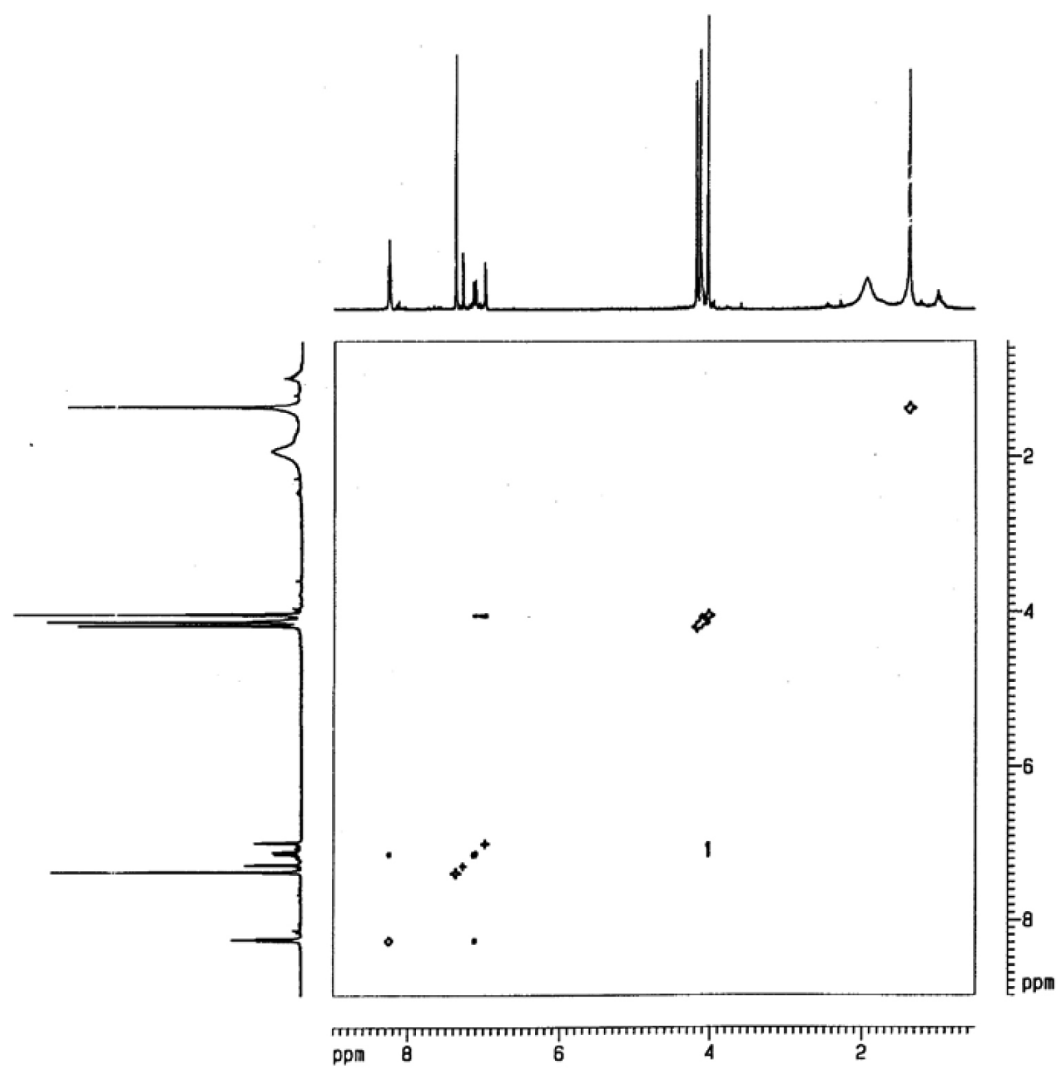

Figure S7. ${ }^{1} \mathrm{H},{ }^{1} \mathrm{H} \quad \mathrm{NOESY}-\mathrm{NMR}$ spectrum of $\mathbf{1}\left(500 \mathrm{MHz}, \mathrm{CDCl}_{3}\right)$. 


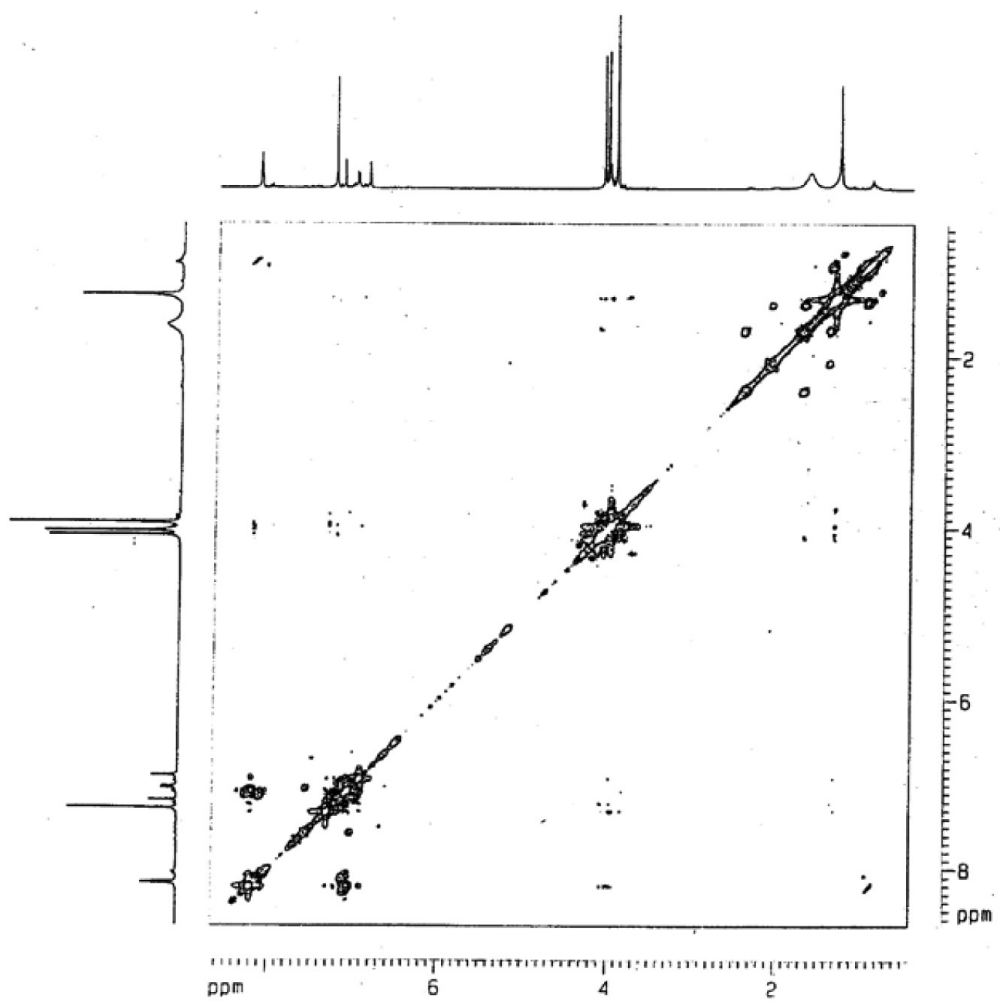

Figure S8. ${ }^{1} \mathrm{H},{ }^{1} \mathrm{H}$ COSY-NMR spectrum of $\mathbf{1}\left(500 \mathrm{MHz}, \mathrm{CDCl}_{3}\right)$.

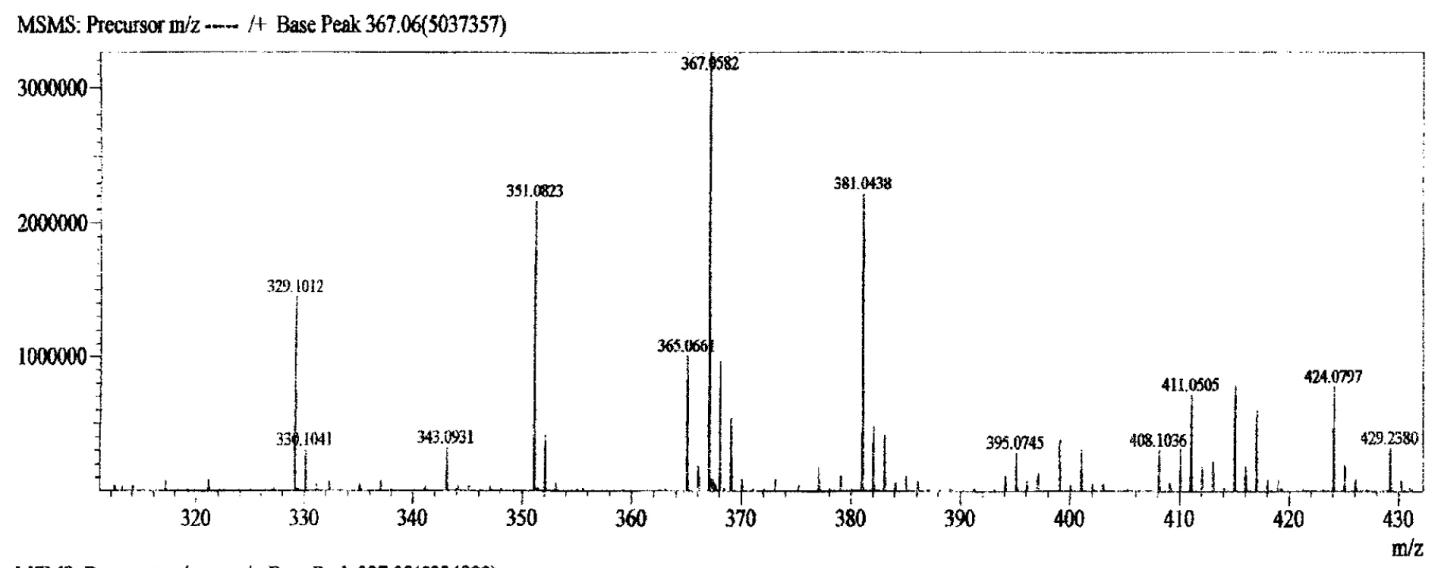

MSMS: Precursor m/z - - Base Peak 327.08(5334983)

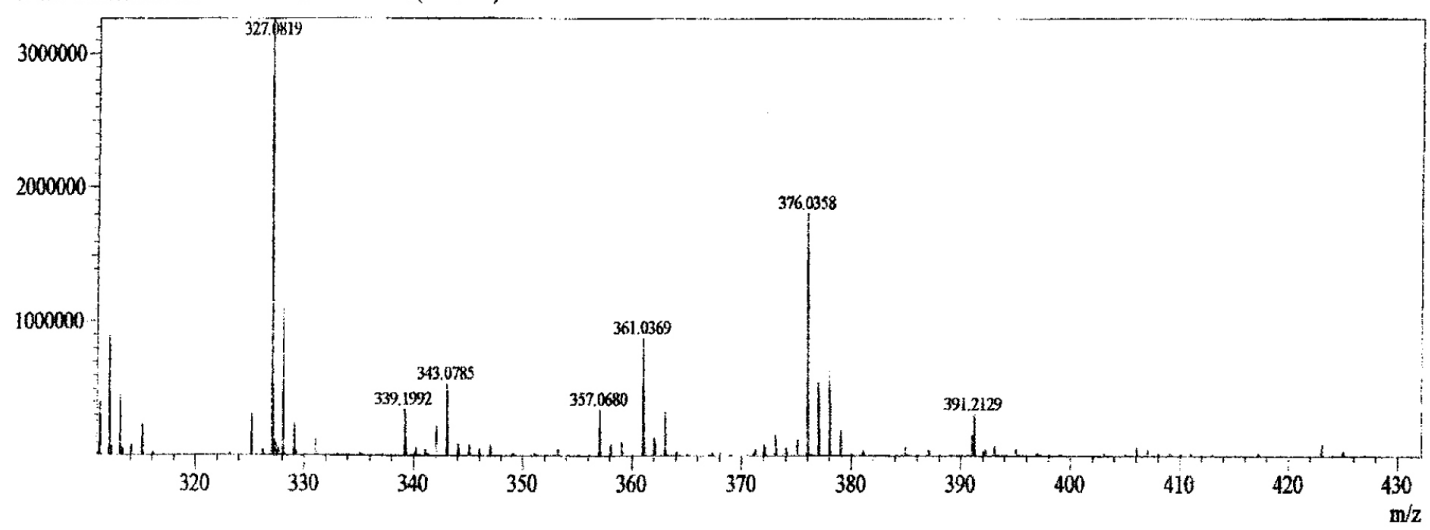

Figure S9. High resolution electrospray ionization mass spectrum of $\mathbf{1}$. 


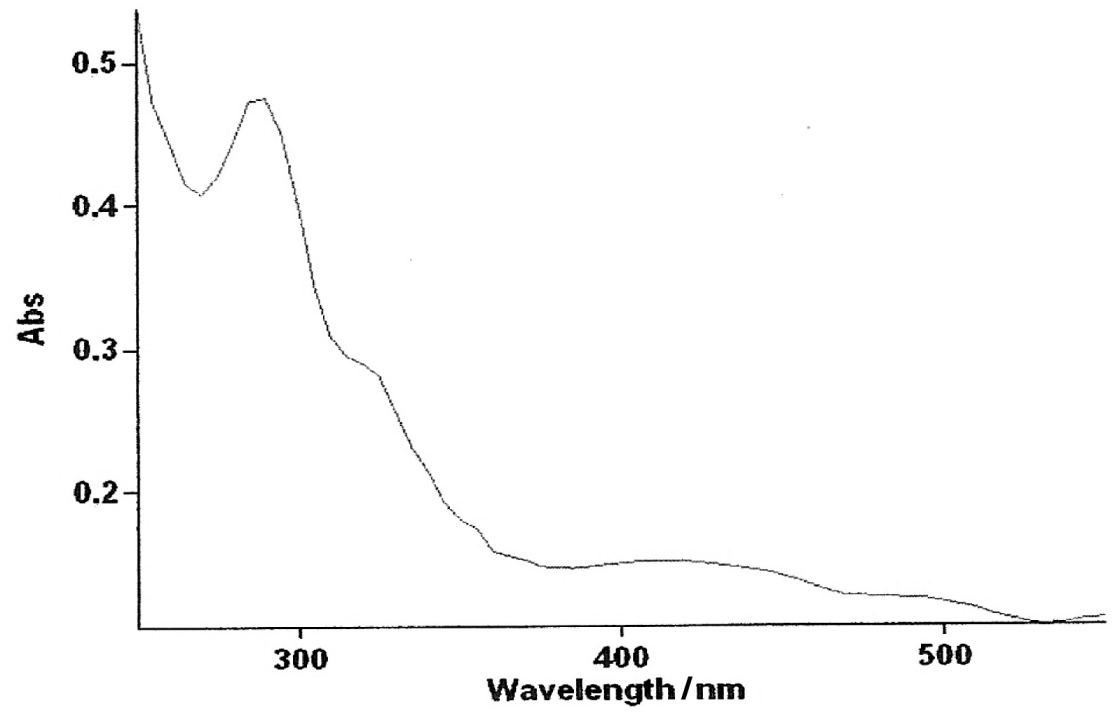

Figure S10. Ultraviolet spectrum of compound $1\left(\mathrm{CH}_{2} \mathrm{Cl}_{2}\right)$. 